\title{
FRACTURING, ACOUSTIC EMISSION, AND NUMERICAL SIMULATION OF GRANITE UNDER MODE II LOADING
}

\author{
O. Stephansson ${ }^{1}$, S. Stanchits ${ }^{1}$, T. Backers ${ }^{1+2}$, G. Dresen ${ }^{1}$ and B. Shen ${ }^{3}$ \\ ${ }^{1}$ GeoForschungsZentrum GFZ Potsdam, Germany \\ ${ }^{2}$ GeoFrames, Germany \\ ${ }^{3}$ CSRIO, Australia
}

\begin{abstract}
Mode II loading of drill core samples of medium-grained granite is conducted using the Punch-Through Shear (PTS-) test. Cylindrical samples with circular notches at both end surfaces are subjected to independent confining pressure and shear loading of the intact portion between the notches. This paper presents results from PTS- testing at confining pressure of $30 \mathrm{MPa}$, including the pressure dependency of Mode II fracture toughness, $\mathrm{K}_{\mathrm{IIC}}$, the resulting fracture pattern, acoustic emission (AE) characteristics and computer simulation. At failure a shear fracture connects the upper and lower notch. Fracture evolution on the macroscopic scale is described. Analysis of AE and micro-structural observations show contribution of both tensile and shear cracks to fracture propagation. AE polarity analysis shows a shift from dominantly tensile cracking to shear cracking during increasing punch loading. This suggests that fracturing in the PTS- test involves mixed-mode fracturing. The fracture initiation and propagation is analysed using the displacement discontinuity code FRACOD ${ }^{2 \mathrm{D}}$. The code simulates the fracture initiation and propagation and acoustic emission of the PTS- test with a fair agreement with the experimental results.
\end{abstract}

\section{INTRODUCTION}

A new method for determination of the critical stress intensity factor in Mode II, the PunchThrough Shear (PTS-) test, was introduced by Backers [1]. The unique feature of the PTS- test is the ability to apply a confining pressure independent of the Mode II (shearing) load. This contribution reports the determined Mode II fracture toughness, $\mathrm{K}_{\mathrm{IIC}}$, and its dependency on confining pressure for Mizunami granite, and examines the AE (acoustic emission) activity and fracture content of the Mode II fracture. A two-dimensional fracture propagation code, FRACOD $^{2 \mathrm{D}}$, has been applied to simulate fracture initiation and fracture propagation in PTSsamples. The results from modelling are compared to the resulting fracture pattern and AE recordings.

\section{EXPERIMENTAL SETUP}

The Punch-Through Shear (PTS-) test uses cylindrical samples with circular notches drilled centred into the end surfaces. The notches serve as friction free initial fractures. The experimental assembly is given in Figure 1. A stiff servo-controlled (MTS) loading machine is used for axial loading. The inner cylinder of the sample is punched down at constant displacement rate $\left(3.3 \cdot 10^{-5} \mathrm{~m} / \mathrm{s}\right)$ until failure, generating a localised shear stress between upper and lower notch. The applied confining pressure generates a normal stress on the shear fracture. Details on the PunchThrough Shear test are given in Backers et al. [1].

The acoustic monitoring system consists of eleven piezoelectric transducers glued to the sample surface. During testing, ultrasonic transmission tests are performed periodically to monitor P-wave velocities in different directions. Hypocenter location is determined by a least square iterative technique to an accuracy of $\sim 2 \mathrm{~mm}$. For details on the recording system and location analysis see Zang [2]. 
(A)

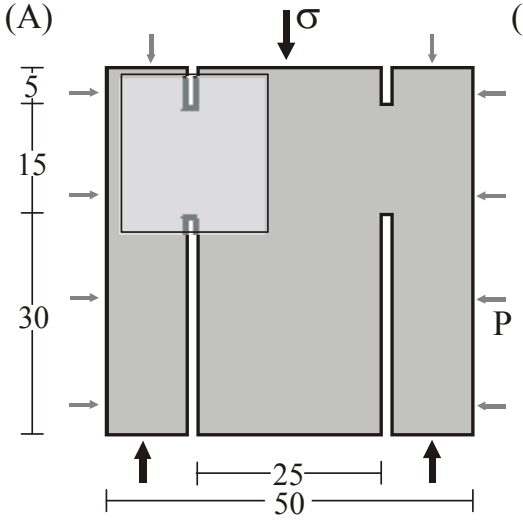

(B)

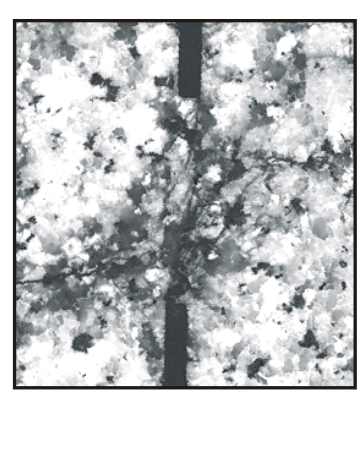

(C)

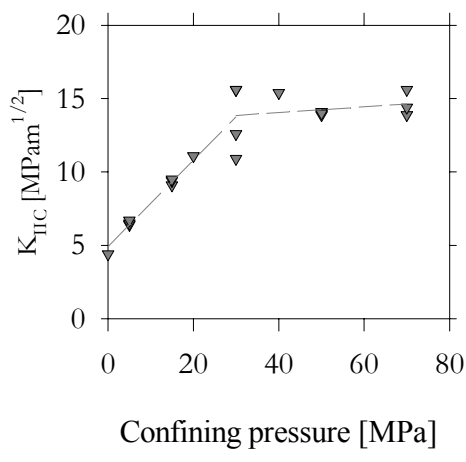

Figure 1: (A) Sample geometry, principle loading, and dimension for the PTS-Test in [mm]. (B) Cross cut view of the granite sample in the area between the notches as highlighted by the light grey area in (A). (C) $\mathrm{K}_{\mathrm{IIC}}$ as function of the confining pressure, $\mathrm{P}$, for Mizunami granite.

\section{EXPERIMENTAL RESULTS}

\subsection{Fracture toughness and fracture content}

The PTS- test is currently performed at confining pressures, $\mathrm{P}$, up to $70 \mathrm{MPa}$. $\mathrm{K}_{\mathrm{IIC}}$ increases nonlinear with P (Fig. 1). Transition from steep to shallow slope is at about 25-35 MPa. This was reported for several rock types (c.f. Backers et al. [3]). $\mathrm{K}_{\mathrm{IIC}}$ of Mizunami granite rises from $\sim 4.9 \mathrm{MPa} \mathrm{m}^{1 / 2}$ at $\mathrm{P}=0 \mathrm{MPa}$ to $\sim 14.9 \mathrm{MPa} \mathrm{m}^{1 / 2}$ at $\mathrm{P}=30 \mathrm{MPa}$, and shows $\sim 15.1 \mathrm{MPa} \mathrm{m}^{1 / 2}$ at $\mathrm{P}=$ $70 \mathrm{MPa}$.

The granite develops a wide process zone that is initiated at about $30 \%$ of the maximum load starting at the bottom notch at $\mathrm{P}=30 \mathrm{MPa}$. In this intensely microcracked zone the main fracture develops and connects the notches at the peak load (Fig. 1). The rock develops a network of predominately grain boundary but also intragranular cracks. Typically features show the direction of en échelon fracturing (Backers et al. [4]).

\subsection{Acoustic Emission}

The fracture evolution for a sample loaded at $\mathrm{P}=30 \mathrm{MPa}$ as observed by $\mathrm{AE}$ analysis is shown in Figure 2.A+B. Continuous AE activity starts at about $45 \%$ of the failure load. The located events form clusters at top and bottom notch. With increase in axial load the bottom cluster starts to propagate upwards with approximately constant speed. The length of the AE process zone increases $(\sim 5-6 \mathrm{~mm})$. The top cluster remains stationary (length: $\sim 2-3 \mathrm{~mm})$. After failure the events are evenly distributed between the notches at lower rate. For further details about the AE monitoring see Stanchits et al. [5].

AE first motion polarities were used to separate the signals into tensile (T-type), shear (S) and pore collapse (C) sources. Spatial distributions of T-S-C types of AE events at different stages of loading are presented in Fig. 2.C. At the initial loading $\left(t_{1}\right)$ equal amounts of T-, S- and C- type events are evident. Once the bottom cluster has started to move upwards $\left(t_{2}\right)$, the relative amount of T- type events is lowered at bottom and top notch whilst the relative amount of C-type events is 
A
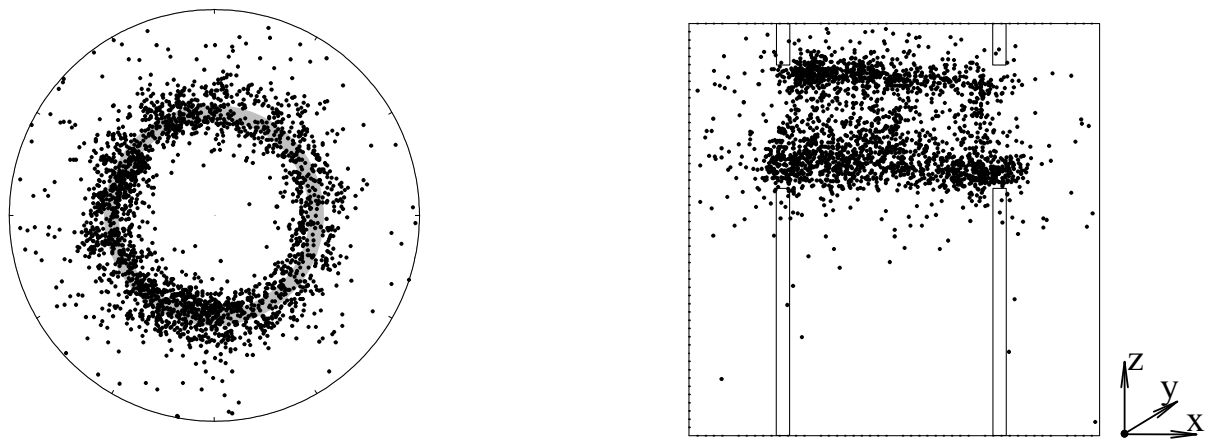

$\mathrm{B}$
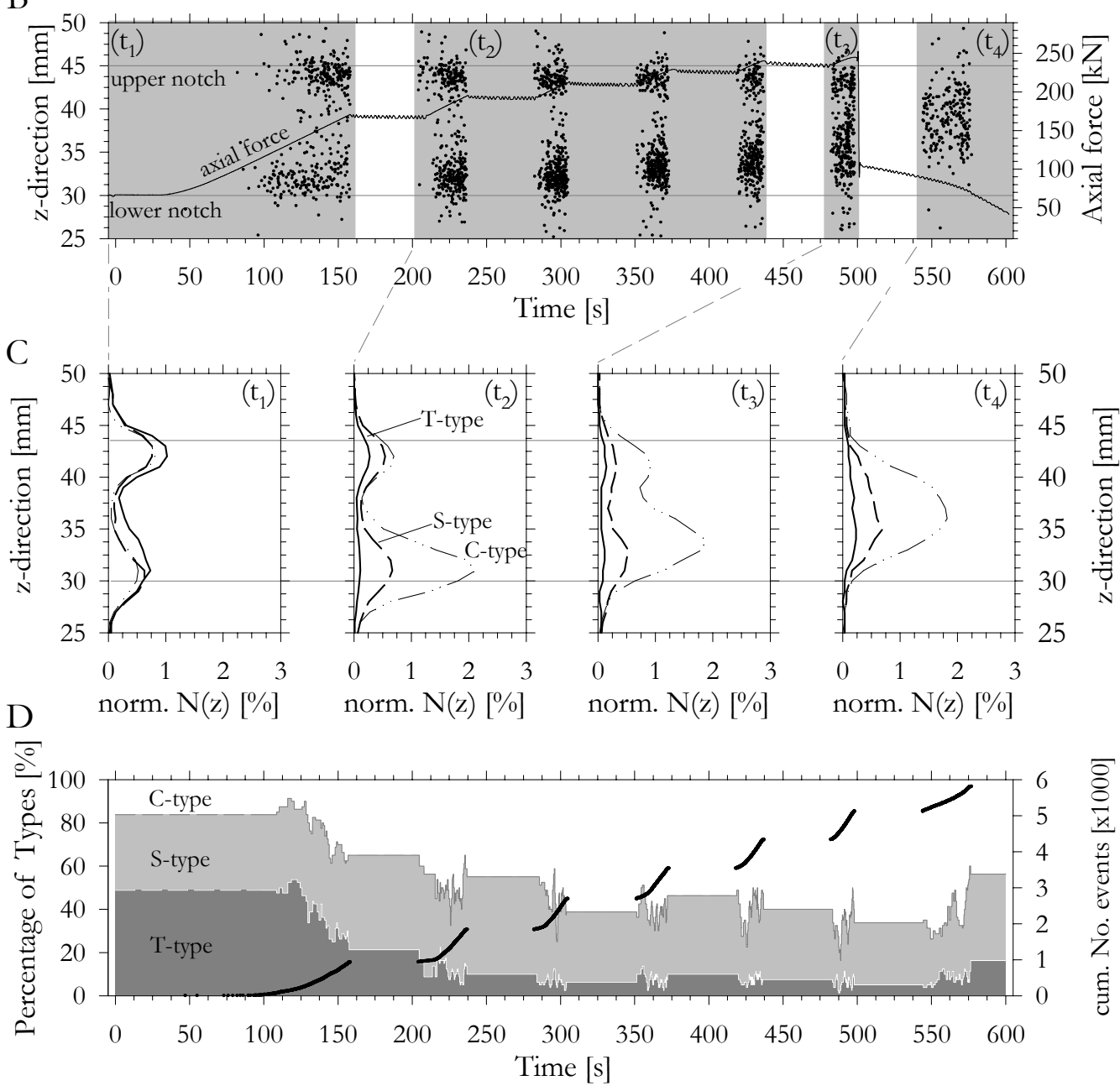

Figure 2: Acoustic Emission (AE) events in space and time. (A) AE distribution in map and side view. (B) The AE events are projected into z-direction and a force vs. time plot are given. (C) Polarity analysis in time slices as indicated in (A). (D) Time distribution of the Polarity distribution and cumulative AE number. 
increased. The relative amount of S-type events remains constant. This picture is valid close to peak load ( $\left.\mathrm{t}_{3}\right)$ also. Post-peak the activity is lowered, but $\mathrm{S}$ - and C- type events are the dominant ones still.

\section{MODELLING OF PTS TEST}

FRACOD $^{2 D}$ (Shen [6]) is a two-dimensional code which is based on the Displacement Discontinuity Method (DDM) principles. It predicts the explicit fracturing process including fracture sliding/opening, fracture initiation and fracture propagation in rocks based on the Fcriterion (Shen and Stephansson [7]). According to the F-criterion, in an arbitrary direction, $\theta$, at a fracture tip there exists a F-value, which is calculated by

$F(\theta)=\frac{G_{I}(\theta)}{G_{I c}}+\frac{G_{I I}(\theta)}{G_{I I c}}$

where $G_{\text {Ic }}$ and $G_{\text {IIc }}$ are the critical strain energy release rates for Mode I and Mode II fracture propagation; $\mathrm{G}_{\mathrm{I}}(\theta)$ and $\mathrm{G}_{\mathrm{III}}(\theta)$ are strain energy release rates due to the potential Mode I and Mode II fracture growth of a unit length. The direction of fracture propagation is defined as the direction of maximum F. At maximum $\mathrm{F}$ equal to 1.0, fracture propagation occurs.

FRACOD $^{2 \mathrm{D}}$ is applied to simulate the fracturing of the PTS- test of Mizunami granite. Input parameters to the code are: $\mathrm{E}=49.9 \mathrm{GPa}, v=0.37, \mathrm{UCS}=166 \mathrm{MPa}$, tensile strength $=9 \mathrm{MPa}$, cohesion, $\mathrm{c}=9 \mathrm{MPa}$, internal fraction angle, $\varphi=33^{\circ}, \mathrm{K}_{\mathrm{IC}}=2.8 \mathrm{MPa} \mathrm{m}^{1 / 2}, \mathrm{~K}_{\mathrm{IIC}}=15.1 \mathrm{MPa} \mathrm{m}^{1 / 2}$, fracture normal stiffness $\mathrm{k}_{\mathrm{n}}=13800 \mathrm{GPa} / \mathrm{m}$, fracture shear stiffness $\mathrm{k}_{\mathrm{s}}=3099 \mathrm{GPa} / \mathrm{m}$, dilation angle $=0^{\circ}, \mathrm{P}=30 \mathrm{MPa}$ and axial load is $167 \mathrm{MPa}$.

The upper and lower notches are simulated by fractures with low strength and deformability. The fracture development during different stages is shown in Figure 3. First a wing fracture is initiated at the bottom and top notches, but stops. Thereafter, a series of en échelon tensile fractures develops between the notches. Finally a set of shear fractures coalesces the tensile fractures and a main shear fracture is formed.

Fracture propagation as calculated by FRACOD is dominated by tensile fracture propagation in the pre-peak, i.e. pre-notch coalescence phase. Shear is evident only on reactivated segments of the wing fracture. During coalescence of the notches shear is the dominant mode, as the inclined struts formed in Mode I are connected by shear fractures.
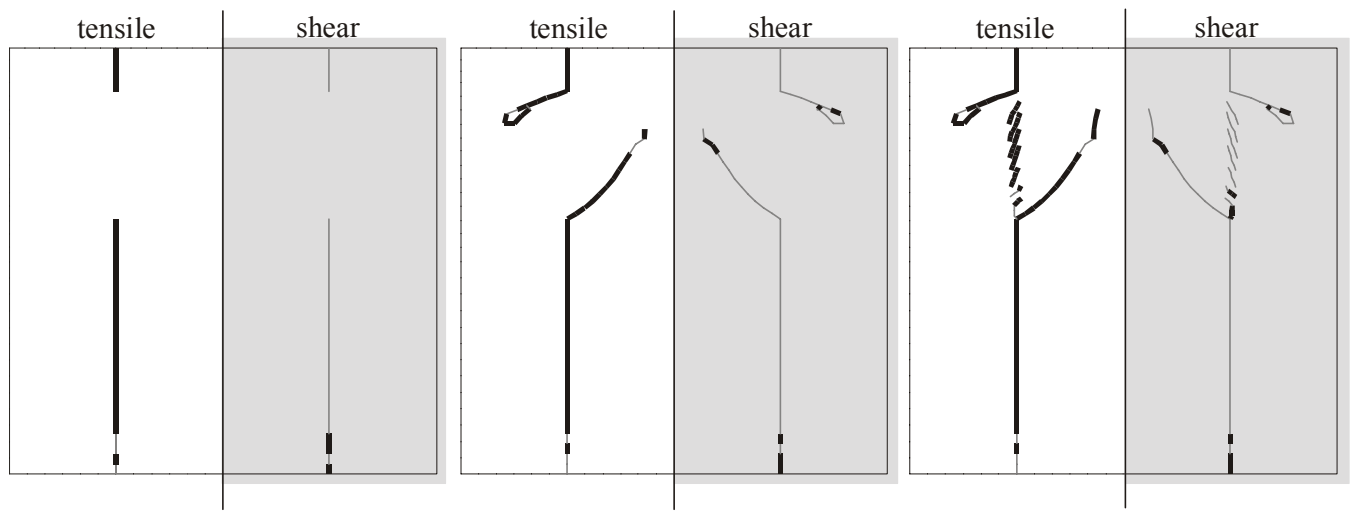

Figure 3. Fracture pattern as predicted from FRACOD modelling at different stages. Left side of each stage snap-shot shows tensile events while right hand side gives segments activated in shear. 


\section{COMPARISON AND DISCUSSION OF EXPERIMENTAL AND MODELLING RESULTS}

The analysis of the fracture pattern and the history of fracturing on samples loaded to different fractions of the peak load gives a consistent picture of the fracture pattern. The shear loading in combination with the confining pressure, i.e. normal stress on the fracture, develops a wide zone of microcracking which connects to a shear fracture at peak load. Increasing the confining pressure increases the fracture toughness, but it does not show a linear increase as was demonstrated for $\mathrm{K}_{\mathrm{IC}}$ (Winter [8]). The bi-linear increase might be dedicated to a change in dominant mechanism in the fracturing process. As $\mathrm{K}_{\mathrm{IC}}$ and $\mathrm{K}_{\mathrm{IIC}}$ show different behaviour to the increase of confining pressure (Figure 4), presumably the Mode I fracturing on the microscale is increasingly suppressed leading to 'purified' Mode II fracture propagation at elevated confining pressure.

The location analysis of the AE gives a very good description of the spatial distribution and history of the fracturing process. Despite the limited spatial resolution of about $2 \mathrm{~mm}$ the physical fracture pattern is very nicely mirrored by the AE. The polarity analysis indicates a distinct contribution of tensile dominated fracturing (T-type) at the notches on progressive loading. The relative amount of T-type events becomes smaller once the fracture starts propagating close to peak load. Hence it may be concluded that the tensile dominated events are predominately related to the formation of the early process zone. Increased shear load results in a shift to shear (S-type) and most prominently pore collapse (C-type) events. During the formation of the matured fracture process zone and the onset of fracture propagation more and more shearing and related pore collapse takes over, resulting in presumably shear enhanced compaction during the connection of the notches. Here C-type events contribute to more than $60 \%$. The total contribution of the tensile dominated fracturing as defined by $\mathrm{AE}$ is very little compared to the S- and C- type events, which can both be linked to the shearing process.

While the fracture pattern and the Acoustic Emissions are both physically measurable, the FRACOD simulation is a simplified model. In contrast to the observed fracture pattern, the numerical modelling results show tensile fracture initiation and the formation of tensile wing fractures at the top and bottom notch, cf. Figure 3. This feature is typically observed in

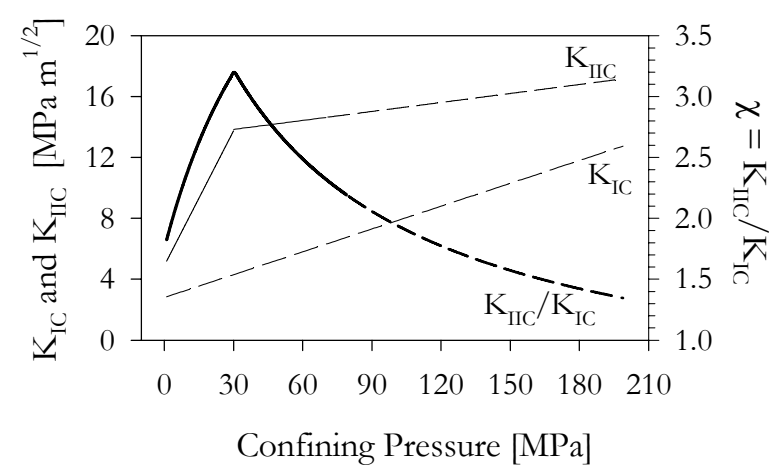

Figure 4. $\mathrm{K}_{\mathrm{IC}}, \mathrm{K}_{\mathrm{IIC}}$ and the ratio $\chi=\mathrm{K}_{\mathrm{IIC}} / \mathrm{K}_{\mathrm{IC}}$ vs. confining pressure, $\mathrm{P}$, for Mizunami granite. Assume $\mathrm{K}_{\mathrm{IC}}=\mathrm{K}_{\mathrm{IC}}^{0}+0.05 \cdot \mathrm{P}$ for the increase of Mode I fracture toughness with increase of confining pressure, as shown by Winter (1983) for Ruhr sandstone up to $\mathrm{P}=100 \mathrm{MPa}$, extension of the linear trend for $\mathrm{K}_{\mathrm{IC}}$ up to higher confining pressures, and low slope increase of $\mathrm{K}_{\text {IIC }}$ above the tested range of confining pressures. Dashed lines indicate assumed data. $\chi$ produces a maximum at a confining pressure of about 25-30 MPa. 
experiments at low confining pressures $(\mathrm{P}<20-30 \mathrm{MPa})$, but not at the given boundary conditions.

As the wing fracture comes to a halt in the numerical model, tensile en èchelon fractures form between the top and bottom notch. The recording of the $\mathrm{AE}$ also depicts this situation. As fracturing within the modelling proceeds the tensile fractures coalesce in shear and a major shear fracture is formed. At this stage the model becomes unstable.

According to the F-criterion as described in Section 4, tensile fracturing should dominate as $\mathrm{G}_{\text {IIC }}$ is in excess of $\mathrm{G}_{\mathrm{IC}}$ or $\mathrm{K}_{\text {IIC }}>\mathrm{K}_{\mathrm{IC}}$. To test the validity of the F-criterion of FRACOD the same model with the same material properties was run but now with $\mathrm{K}_{\mathrm{IIC}}<\mathrm{K}_{\mathrm{IC}}$. The result gives no tensile fracturing and wing crack formation but a straight through-going shear fracture between the upper and lower notch. This situation has also been simulated in PTS testing of porous mortar where $\mathrm{K}_{\mathrm{IIC}}$ is less than $\mathrm{K}_{\mathrm{IC}}$. A sensitivity analysis about the influence of the model parameters has been performed and the results will be presented in the full paper of this contribution.

\section{CONCLUSIONS}

- Shear fracture initiation within the PTS- test are closely associated with formation of up to $50 \%$ of tensile cracks at the beginning of loading suggesting the formation of an initial process zone that is dominated by dilatant cracks.

- Pore collapse events increase at the expense of T-type events during progressive loading. This suggests that during shearing previously formed cracks are compacted.

- FRACOD code simulates the fracture initiation and propagation with a fair correspondence with the experimental results.

\section{REFERENCES}

[1] Backers T., Stephansson O. \& Rybacki E. Rock Fracture Toughness Testing in Mode II Punch-Through Shear Test. Int. J. Rock Mech. Min. Sci, 39: 755-769. 2002.

[2] Zang, A., Wagner, F.C., Stanchits, S., Dresen, G., Andresen, R. \& Haidekker, M. Source analysis of acoustic emissions in Aue granite cores under symmetric and asymmetric compressive loads. Geophysical Journal International; 135: 1113-1130. 1998.

[3] New data

[4] Backers T., Rybacki E., Alber M. \& Stephansson O. Fractography of rock from the new Punch-Through Shear Test. In: Dyskin, A.V., Hu, X. \& Sahouryeh, E. (eds.). Structural Integrity and Fracture - The International Conference on Structural Integrity and Fracture, Perth, Australia: 303-308. 2002.

[5] Stanchits S., Backers T., Stephansson O. \& Dresen G. Comparison of Acoustic Emission Events and Micromechanics of granite under Mode I and Mode II loading. In: Makurat A. \& Curri P. (eds.). EURO-Conference on Rock Physics and Geomechanics - Micromechanics, Flow and Chemical Reactions. Extended Abstract Volume, 7-11 September 2003, Delft, Netherlands. 2003. [6] Shen, B. FRACOD User's manual. Fracom Ltd. 2002.

[7] Shen, B. \& Stephansson, O. Modification of the G-criterion of crack propagation in compression. Int. J. Eng. Fract. Mech.; 47: 177-189. 1993. 